\title{
Phytoprotection
}

\section{La santé des racines : le monde de la complexité}

\section{André Comeau, François Langevin et Marcel Lévesque}

Volume 86, numéro 1, avril 2005

URI : https://id.erudit.org/iderudit/011714ar

DOI : https://doi.org/10.7202/011714ar

Aller au sommaire du numéro

Éditeur(s)

Société de protection des plantes du Québec (SPPQ)

ISSN

0031-9511 (imprimé)

1710-1603 (numérique)

Découvrir la revue

\section{Citer cet article}

Comeau, A., Langevin, F. \& Lévesque, M. (2005). La santé des racines : le monde de la complexité. Phytoprotection, 86(1), 43-52. https://doi.org/10.7202/011714ar

\section{Résumé de l'article}

L'étude des racines a été entreprise dans le laboratoire du Centre de recherche et de développement sur les sols et les grandes cultures pour tenter d'élucider l'effet inattendu d'un virus qui, exploité dans le contexte de la sélection végétale, aidait à augmenter le potentiel de biomasse de l'espèce. La complexité de la vie des racines amène bien des embûches dans de telles études. Le monde des interactions au niveau des racines est si complexe que la méthode cartésienne n'offre pas les outils adéquats pour comprendre le système. Malgré tout, on peut développer des méthodes utiles et efficaces pour comprendre et gérer cette complexité. Après avoir approfondi les acquis des méthodes cartésiennes, il faut aller vers des approches synthétiques offrant une possibilité de continuité des progrès académiques et pratiques. Les racines les mieux adaptées et les plus plastiques par rapport à leur écosystème possèdent de nombreuses propriétés probablement interreliées. Un progrès global vers la santé des racines est donc un but envisageable, et dont le succès serait important au niveau environnemental. La génétique et la régie peuvent y contribuer. Pour progresser dans ce domaine, il faut donc oser sortir des voies conventionnelles. Ce n'est pas seulement la difficulté d'une question complexe qui est en cause, mais également le fait que notre éducation nous a enseigné à éviter de côtoyer la complexité. L'approche réductrice ne permet de comprendre ni la santé des racines, ni celle de la plante, ni le lien entre la plante, ses ennemis, la rhizosphère, et l'environnement. C'est donc à juste titre que des philosophes nous mettent en garde contre les impacts du morcellement des connaissances. Ce texte s'inscrit dans le désir d'aborder le monde des racines dans sa complexité et d'éviter les pièges associés à la logique cartésienne à l'état pur. 


\title{
La santé des racines : le monde de la complexité
}

\author{
André Comeau', François Langevin² et Marcel Lévesque ${ }^{3}$
}

PHYTOPROTECTION 86 : 43-52

\begin{abstract}
L'étude des racines a été entreprise dans le laboratoire du Centre de recherche et de développement sur les sols et les grandes cultures pour tenter d'élucider l'effet inattendu d'un virus qui, exploité dans le contexte de la sélection végétale, aidait à augmenter le potentiel de biomasse de l'espèce. La complexité de la vie des racines amène bien des embûches dans de telles études. Le monde des interactions au niveau des racines est si complexe que la méthode cartésienne n'offre pas les outils adéquats pour comprendre le système. Malgré tout, on peut développer des méthodes utiles et efficaces pour comprendre et gérer cette complexité. Après avoir approfondi les acquis des méthodes cartésiennes, il faut aller vers des approches synthétiques offrant une possibilité de continuité des progrès académiques et pratiques. Les racines les mieux adaptées et les plus plastiques par rapport à leur écosystème possèdent de nombreuses propriétés probablement interreliées. Un progrès global vers la santé des racines est donc un but envisageable, et dont le succès serait important au niveau environnemental. La génétique et la régie peuvent y contribuer. Pour progresser dans ce domaine, il faut donc oser sortir des voies conventionnelles. Ce n'est pas seulement la difficulté d'une question complexe qui est en cause, mais également le fait que notre éducation nous a enseigné à éviter de côtoyer la complexité. L'approche réductrice ne permet de comprendre ni la santé des racines, ni celle de la plante, ni le lien entre la plante, ses ennemis, la rhizosphère et l'environnement. C'est donc à juste titre que des philosophes nous mettent en garde contre les impacts du morcellement des connaissances. Ce texte s'inscrit dans le désir d'aborder le monde des racines dans sa complexité et d'éviter les pièges associés à la logique cartésienne à l'état pur.
\end{abstract}

Mots clés: Descartes, Pascal, photosynthétats, résistance, systémique, tolérance.

\section{[Root health: a world of complexity]}

A root study was undertaken at the Soils and Crops Research and Development Centre laboratory in an attempt to elucidate the unexpected impact of a virus which, used in the context of plant selection, helped improve the biomass potential of the species. The complexity of root systems can produce many pitfalls in such studies. Interactions at the root level are so complex that Cartesian methods do not offer suitable tools for understanding the system. However, it is possible to develop useful and efficient methods for understanding and managing this complexity. After having analyzed the knowledge acquired using Cartesian methods, it is necessary to look into synthetic methods offering the possibility of furthering academic and practical progress. The best adapted and most plastic roots in relation to their ecosystem have numerous characteristics that are probably interrelated. Hence, a global progress towards root health is a realistic objective whose success would be important at the environmental level. Genetics and management also have a role to play. To make progress in this field, it is therefore necessary to dare go off the beaten track. It is not only the difficulty of a complex issue that is in question but also the fact that our education has taught us to avoid meddling with complexity. The simplistic approach does not allow a good understanding of root heath, or even plant health, or of the relationships between the plant and its enemies, the rhizosphere and the environment. Philosophers are therefore justified in warning us against the impacts of knowledge fragmentation. The following text comes within the scope of a desire to tackle the world of roots in all of its complexity in order to avoid the traps inherent to pure Cartesian logic.

Keywords: Descartes, Pascal, photosynthates, resistance, systemic, tolerance.

1. Centre de recherche et de développement sur les sols et les grandes cultures, Agriculture et Agroalimentaire Canada, Sainte-Foy (Québec), Canada G1V 2J3; courriel : comeaua@agr.gc.ca

2. Centre de recherche sur les grains inc. (CÉROM), Saint-Bruno-de-Montarville (Québec), Canada J3V 4P6

3. Institut de recherche et de développement en agroenvironnement, Saint-Hyacinthe (Québec), Canada J2S 7B8 


\section{INTRODUCTION}

La vie des plantes : un lien réciproque entre la partie cachée et la partie visible

La racine est un organe clé des végétaux. Mais la complexité de la rhizosphère résiste à l'analyse, et plusieurs méthodes d'étude des racines sont coûteuses et destructrices. On étudie plus facilement les parties aériennes des plantes. Celles-ci habitent un milieu chimiquement assez simple, majoritairement composé d'oxygène, d'azote, de $\mathrm{CO}_{2}$, de vapeur d'eau et de quelques molécules organiques. La composition de l'atmosphère varie peu autour de notre planète. Ajoutons les diverses radiations et la température, et tout le monde aérien abiotique a été décrit. Bien sûr, les maladies et les insectes compliquent le portrait, mais on n'a pas besoin de creuser pour constater l'état des feuilles, des tiges, des fleurs et des fruits.

Tous les minéraux de l'ensemble de la plante ont transité par les racines. Or, sous terre, le monde rencontré par la plante varie sans cesse dans l'espace et le temps. La nature de la roche-mère et le climat sont les premiers facteurs qui définissent l'évolution du sol et le milieu de vie des racines et de toute la vie qui l'entoure. La pédogenèse implique également l'action des plantes et des microorganismes. L'environnement physique et chimique diffère beaucoup entre la surface et les profondeurs du sol. La plante interagit avec une bonne partie des éléments plus légers du tableau périodique de Mendeleïev. Parmi les 30 premiers éléments du tableau, les plantes en utilisent de manière spécifique de 18 à 21 . Le sodium, I'aluminium, le silicium et le nickel ne jouent pas un rôle universel, mais sont aptes à favoriser la croissance et la santé de certaines espèces (Marchner 1995). La complexité minérale du milieu souterrain augmente le défi d'apporter aux cellules ce dont elles ont besoin, tout en éliminant les formes toxiques, à travers les variations de température, d'humidité et de potentiel redox. Une multitude de mécanismes flexibles sont nécessaires, et ces mécanismes doivent être régulés. Par ailleurs, le potentiel redox, qui est fonction de l'oxygène, est un facteur de stress important pour bien des cultures. Les plantes doivent donc fonctionner avec l'oxygène qui se rend aux racines soit à travers le sol, soit à travers l'aérenchyme qui est un tissu spécialisé qui véhicule l'oxygène dans les racines. Selon le potentiel redox, on constate la formation d'ions dans un état plus ou moins oxydé; la microflore anaérobique s'installe aux potentiels plus bas, et un grand nombre de substances toxiques se forment (Kozlowski 1984). Avant de considérer les facteurs biotiques, il faut donc avoir pris conscience de la complexité de base au niveau minéral, hydrique, et potentiel redox. II faut encore assimiler le fait que les espèces qui gravitent autour de la rhizosphère (bactéries, champignons, nématodes, insectes, etc.) sont également sujettes aux effets de la constitution minérale du milieu. Selon Browning (1998), on enseigne la pathologie des plantes, mais il faudrait réorienter l'enseignement vers la santé des plantes et la compréhension des interactions.

\section{PROBLÉMATIQUE}

Réapprendre à penser

Pourquoi a-t-on aussi peu favorisé les recherches sur les racines, est-ce seulement parce que c'est difficile? Plusieurs penseurs ont noté combien l'esprit humain affectionne les raisonnements et conclusions simples. Descartes, avec son discours sur la méthode, a d'ailleurs poussé dans cette direction plusieurs générations successives de penseurs et de scientifiques. C'est de Descartes et de ses disciples que provient la notion qu'on peut découvrir dans la nature l'ordre et la stabilité, qu'on peut identifier les causes séparées des phénomènes et, qu'ayant compris individuellement ces liens de causalité, on peut alors arriver à comprendre le tout, à partir du moment où on a bien compris toutes les causalités entre les parties. C'est à partir d'une telle philosophie que certains ont encore pu rêver, il y a six ans à peine, que le vivant était comme une machine et qu'il suffisait de décoder I'ADN pour découvrir la base qui permet de tout comprendre. On oubliait alors le cross-talk, la rétroaction et les phénomènes chaotiques mathématiquement inévitables puisque le vivant existe grâce aux phénomènes récurrents et répétitifs. On sous-estimait également tous les niveaux supérieurs $d^{\prime}$ interactions complexes entre I'ADN, l'ARN, les protéines, les autres composantes cellulaires, base d'un réseau de communication bidirectionnelle avec l'environnement interne et externe.

Un exemple des faiblesses de l'approche cartésienne semble à propos. Le blé peut souffrir de plus d'une cinquantaine de stress différents (Comeau et al. 2001). Plus de la moitié de ces stress sont liés aux racines. Comme l'intensité, la durée et la fréquence peuvent varier pour chaque stress, et que les stress peuvent interagir entre eux, il devient impossible de dénombrer par l'approche cartésienne la diversité des environnements stressants possibles. Nous voulons ainsi souligner l'impossibilité pratique d'une approche cartésienne à un sujet complexe, ce qui nous mène à citer Blaise Pascal (cité dans Morin 1999) : " Je tiens impossible de connaître les parties sans connaître le tout, non plus que de connaître le tout sans connaître particulièrement les parties ". Cet énoncé n'est pas facile à assimiler tant que nous nous restreignons à la logique cartésienne. II faut comprendre qu'un système possède ses propres règles, et que les éléments de causalité linéaire ne suffisent pas. Par ailleurs, plusieurs livres de vulgarisation écrits par Richard Lewontin peuvent nous aider à entrer dans la voie de la pensée systémique (Lewontin 1991, 2000, 2001; Lewontin et al. 1984). Pour comprendre le monde des racines, être chercheur ne suffit pas; il faut vraiment devenir penseur.

Pour couvrir convenablement le sujet, il faudrait toucher à de nombreuses disciplines : du côté de I'environnement, chimie et physique des sols, climatologie, écologie; et du côté de la plante, génétique, mécanique, physique, biochimie, physiologie, pathologie, entomologie, nématologie. Enfin, pour en arriver à réfléchir sur la conception d'une d'approche intellectuelle, on n'échappera pas à un retour à la philosophie. Nous chercherons donc des modes $d^{\prime}$ analyse et $d$ 'expérimentation qui intègrent toutes 
les facettes de la santé des racines, sans négliger un questionnement sur l'adaptation des outils statistiques aux sujets complexes.

\section{L'approche systémique}

Étant donné la complexité du sujet, quelques exemples seront abordés afin de mieux comprendre l'approche systémique préconisée par Pascal. II faut étudier à la fois les parties et le tout, et ce, de manière séquentielle ou simultanée. Notre compréhension du système augmente ainsi, mais Lewontin nous aura mis en garde contre l'illusion de pouvoir comprendre de manière exhaustive un sujet complexe.

Les sols de diverses régions dépendent d'abord de la roche-mère et des origines, et sont plus ou moins modifiés par le climat et par les pratiques culturales. La pédogenèse inclut la formation de matière organique et d'acides humiques capables de modifier la disponibilité de l'eau et des minéraux pour la plante (Dommergues et Mangenot 1970). Plusieurs sols du nord-est de l'Amérique sont lessivés et compactés. Les minéraux essentiels sont présents sous plusieurs formes dont certaines sont peu disponibles; le phosphore, le manganèse, le fer et le cuivre peuvent ainsi être présents surtout sous des formes insolubles. Ces réserves importantes de minéraux moins solubles expliquent que les espèces végétales plus efficaces dans la dissolution des formes réfractaires sont très compétitives dans leur milieu naturel et peuvent parfois devenir des mauvaises herbes redoutables. L'amélioration génétique peut arriver à créer des plantes agricoles qui soient aptes à utiliser ces minéraux et nous l'avons démontré récemment en créant un blé qui absorbe efficacement le manganèse dans les sols les plus carencés. Nos travaux connexes sur l'efficacité d'absorption du phosphore, du bore et du cuivre rencontrent également un certain succès. Parmi les autres facteurs de pertes économiques importants associés aux sols, on inclut en général le déficit hydrique; au Québec, il faut ajouter l'excès d'eau et les problèmes d'égouttement (Brunelle 2005). Ce sont là des facteurs auxquels la phytogénétique et les méthodes de culture peuvent remédier.

Nous ne voulons pas entrer ici dans une analyse trop détaillée des problèmes causés par chaque stress biotique et abiotique. Nous ciblons une vision globale du problème et des manières de $s^{\prime} y$ attaquer, non pas en pathologistes, entomologistes, spécialistes en fertilité ou en d'autres domaines, mais plutôt en experts en systèmes, qui recherchent une compréhension plus globale de la santé de la plante. Browning (1998) déclare qu'on sous-estime les facteurs de pertes et leurs interactions et que l'on devrait développer une vision d'ensemble des facteurs. En 1999, nous avons donc conduit une enquête auprès de plusieurs spécialistes en céréales au Canada et en additionnant les divers taux de pertes évalués, on arrivait à des pertes totales d'environ $70 \%$. Un peu plus de la moitié des facteurs de pertes touchaient les racines, et les facteurs abiotiques dominaient, mais de peu. Vers la même époque, Browning (1998) déclarait que, par rapport à leur rendement potentiel, la majorité des plantes agricoles subissaient des pertes totales de 80 à $85 \%$ par la somme des facteurs biotiques et abiotiques, et que la proportion entre ces deux groupes était environ moitié-moitié. Ce ne sont que des estimations, mais elles sont appuyées par la connaissance de rendements records observés en conditions idéales. Par exemple, chez le blé, on a vu des rendements en grains de $18 \mathrm{t} \mathrm{ha}^{-1}$ au Chili, et de plus de $10 \mathrm{t} \mathrm{ha}^{-1}$ pour un blé de printemps au nord de I'Alberta. La différence entre ces rendements exceptionnels et le rendement ordinaire au champ amène la preuve indirecte de l'énormité des pertes.

\section{Liens entre les racines et les parties aériennes}

Notre propre expérience de recherche porte principalement sur les céréales à paille. Il est logique de considérer d'abord les aspects globaux avant les détails. Tout d'abord, MacKey $(1973,1984)$ a observé que, chez les céréales, les plantes plus hautes ont souvent des racines plus longues. Or, la domestication du végétal passe par un raccourcissement, sauf si la tige est très forte comme chez le maïs. Si MacKey a raison, tous ceux qui ont domestiqué et amélioré les plantes auraient modifié accidentellement la zone explorée par les racines. Par l'usage moderne des engrais, on a mis l'emphase sur l'exploration d'un horizon de surface enrichi. À partir du moment où la sélection génétique est effectuée dans des champs cultivés et gérés avec engrais, les sélectionneurs choisissent peut-être automatiquement des plantes dont les racines sont plus superficielles. Quelques informations semblent contradictoires. Par exemple, chez le blé Maringa, dont les racines sont très tolérantes aux stress abiotiques, les gènes de raccourcissement de la paille n'ont pas un effet très marqué sur les racines (Miralles et al. 1997). Néanmoins, nos travaux effectués avec les blés semi-nains associés à la "révolution verte " ont montré que la plupart avaient de sérieux problèmes d'enracinement en présence d'un virus (jaunisse nanisante, ou BYD) ou de stress abiotiques comme les carences, l'acidité ou I'hypoxie (carence en oxygène) (Comeau et Haber 2002). Et même si, à l'intérieur d'une seule espèce, la corrélation entre la longueur des parties aériennes et celle des racines peut sembler faible, cette même corrélation devient indiscutable dans un ensemble plus grand comprenant plusieurs espèces céréalières (Nodichao 1997).

\section{Résistance, tolérance : \\ des notions relatives et non absolues}

Comme les pertes sont aussi importantes, I'hypothèse souvent retenue est que les facteurs de résistance aux stress biotiques et abiotiques apporteront la solution au problème. On choisit alors d'ignorer la différence entre la résistance et la tolérance et d'oublier que ces réponses face aux stress représentent des coûts énergétiques. Or, tout mécanisme de résistance et de tolérance implique une déviation des produits de la photosynthèse vers des fins spécifiques, ce qui représente donc un coût énergétique (Brown 2002; Geiger et Servaites 1991). De plus, la régulation de tels mécanismes est à la fois essentielle et complexe, car ces mécanismes ne devraient idéalement dépenser de l'énergie qu'en cas de besoin, ce qui n'est pas un objectif facile car, pour y arriver, la plante devrait répondre de manière parfaite à de multiples signaux de l'environnement. 
Précisons notre terminologie : la résistance signifie que la plante combat un stress, ou réprime la multiplication et la propagation d'un agent pathogène, alors que la tolérance signifie que la plante subit peu ou pas de pertes même si un agent pathogène ou un stress se développe. Cette seconde proposition semble parfois violer le postulat de Koch, mais elle est aujourd'hui bien démontrée (Comeau et Haber 2002, 2004). Le compromis visé est d'avoir une plante chez laquelle les coûts de résistance et tolérance sont moindres que ceux des pertes évitées. Soulignons que tolérance est synonyme d'efficacité, car si les coûts énergétiques sont élevés, un mécanisme ne remplit pas la définition de tolérance qui spécifie bien "peu ou pas de pertes".

Le terme "tolérance" n'a de sens qu'en fonction d'un environnement donné. Des gènes ou des traits peuvent conférer la tolérance dans un environnement et s'avérer inutiles ou nuisibles dans un autre. Un gène stimulant les exsudats qui solubilisent les minéraux sera très utile en sol pauvre, mais potentiellement consommateur d'énergie en sol riche si l'exsudation n'est pas réduite.

Les tolérances existent et sont en général le choix logique contre le BYD (Comeau et Haber 2002) et souvent aussi contre les maladies des racines (Higginbotham et al. 2004; Nicol et al. 2004).

L'approche systémique que nous avons adoptée nous incite à vérifier les effets dans leur ensemble, donc à l'intérieur d'un système naturel ou complexe. Nous avons innové en ce domaine, en groupant des facteurs de stress et en comparant ces facteurs aux effets de situations de stress naturels. Nous avons également découvert l'utilité des stress comme le virus de la jaunisse nanisante. L'infection a des effets majeurs sur les apex des racines et induit indirecte- ment une multitude de stress chroniques différents chez les céréales. L'usage de tels stress en sélection aide à déceler quelles sont, à travers une population, les plantes qui intègrent plusieurs facteurs de résistance et de tolérance de manière efficace au niveau énergétique (Comeau et al. 2001).

\section{Étude de cas exemplaires}

La citation de Pascal ne nous dit pas de négliger les causalités simples, mais simplement de les relier au système. Prenons le cas de la compétition des plantes cultivées envers les mauvaises herbes. L'outil sélectif que représente I'herbicide fait que les cultures n'ont plus à offrir de compétition envers les mauvaises herbes et peuvent donc optimiser autrement l'usage de l'énergie et des nutriments. Lors d'un processus de sélection génétique en régie avec herbicides, les génotypes dont une partie de l'énergie se dépense en mécanismes de compétition envers les mauvaises herbes seront moins conservés que ceux qui mettent davantage d'énergie en production de grains. Par ailleurs, on trouve chez les blés modernes une dépendance vis-à-vis l'environnement favorable fourni par les engrais, herbicides et autres intrants chimiques (Lemerle et al. 1996). Lorsque l'on compare les anciens cultivars de blé canadien au germoplasme le plus récent, on arrive à peu près aux mêmes conclusions que Lemerle. Néanmoins, l'ancienneté n'est aucunement un gage de compétitivité. Sous conditions de stress, le vieux cultivar de blé Huron (1896) se tire un peu mieux d'affaire face aux mauvaises herbes que d'autres vieux cultivars comme le blé Marquis (1923) qui présente de sérieux problèmes d'adaptation aux stress. Or la plupart des cultivars de I'Ouest sont apparentés au blé Marquis et se comportent comme ce dernier. Par contre, certains cultivars modernes de l'Est du Canada ont une meilleure vigueur compétitrice que le blé Huron. De nouveaux

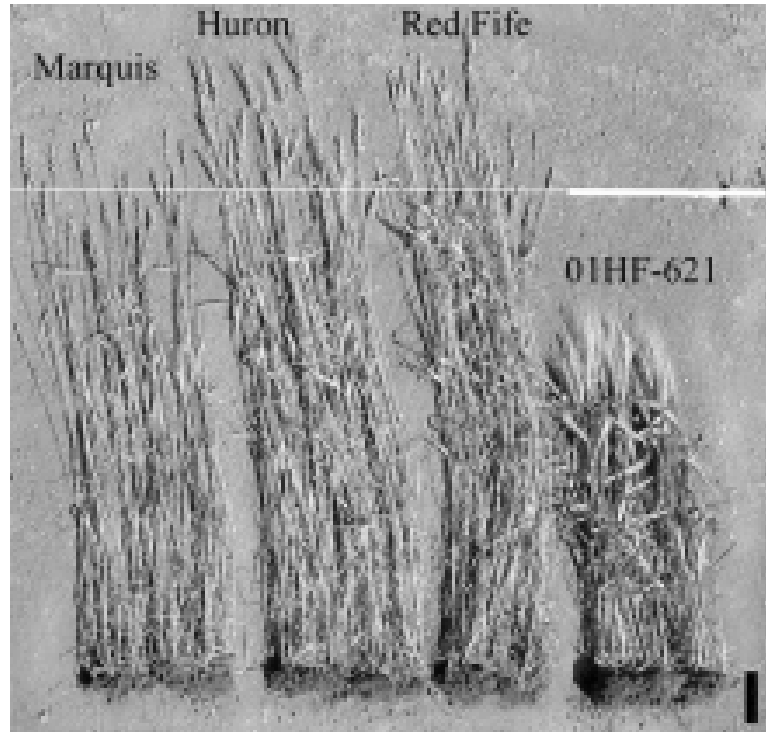

Figure 1. Prélèvement d'environ $30 \mathrm{~cm}$ de rang de quatre lignées de blé cultivés sur une argile assez riche. Ces blés ont subi un certain stress d'excès de pluie et de virus (BYD). Ce sont les cultivars anciens Red Fife (1842), Huron (1896) et Marquis (1923), ainsi que la lignée récente et tolérante au virus $01 \mathrm{HF}-621$, dans un essai inoculé artificiellement de BYD au stade du tallage, en 2004. Noter bien l'épaisseur du sol retenu par les racines. La ligne pâle horizontale est à $1 \mathrm{~m}$ du collet; le losange noir en bas à droite mesure $10 \mathrm{~cm}$ de haut. 


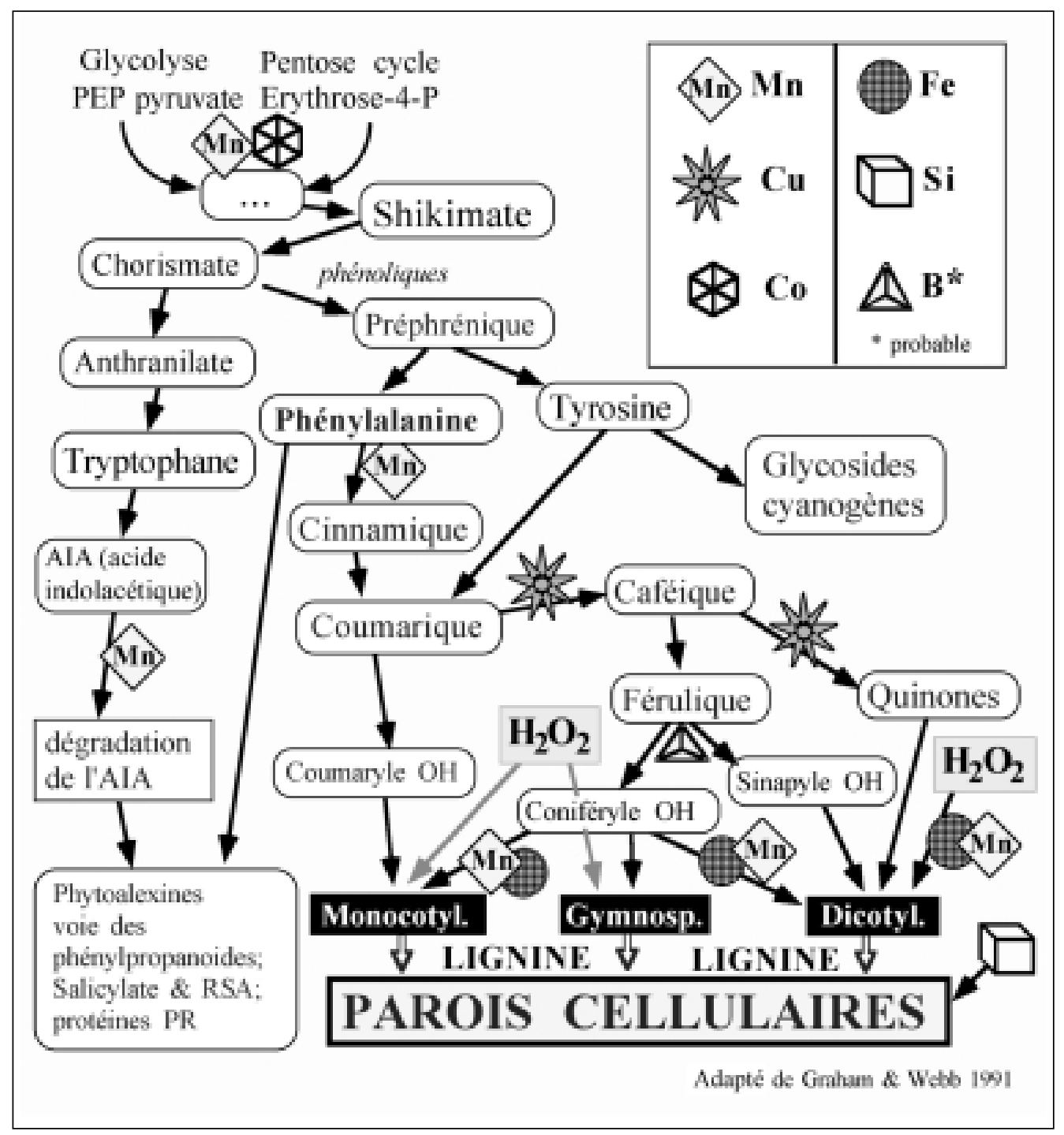

Figure 2. Illustration des nombreux liens entre les oligo-éléments et le sentier métabolique du shikimate, lequel est au coeur de nombreux mécanismes de défense de la plante et essentiel à la formation de la lignine.

blés développés par sélection sous stress complexe, et à base de germoplasme brésilien, tel le croisement 01HF-621, se comportent très bien face aux mauvaises herbes même s'ils sont nains. Ces blés tallent abondamment, produisent beaucoup de racines et sont peu sensibles au stress d'hypoxie, de virus et de sécheresse (Fig. 1). Les racines abondantes aident au remplissage du grain et offrent un meilleur ancrage qui réduit la verse, tel que démontré au Brésil (Cruz et al. 2001).

Un examen plus détaillé de ce progrès s'impose. Graham et Webb (1991) ont fait ressortir le rôle de certains oligo-éléments qui sont essentiels dans les sentiers métaboliques reliés à plusieurs mécanismes de résistance aux ennemis des plantes, ainsi que pour la formation de la lignine et des parois cellulaires (Fig. 2). Par ailleurs, le bore est essentiel pour le transport des sucres et l'élongation des racines (Abdel Nasser et Abdel Aal 2002). II en ressort qu'on ne devrait jamais étudier les mécanismes de résistance aux stress biotiques sans avoir souci du statut nutritionnel des plantes. Avouons que même après avoir lu Graham et Webb, nous avions continué nos travaux sur le BYD sans comprendre immédiatement l'importance réelle de la nutrition minérale. Mais les liens de la tolérance au BYD avec l'efficacité d'absorption des minéraux sont devenus graduellement de plus en plus évidents. Ainsi, une de nos lignées de blé les plus tolérantes aux stress conjoints de virus, d'hypoxie et de maladies des racines, la Mu2EL6Hat, dérivée du rétrocroisement blé//Leymus multicaulis/blé, a été observée comme la plus efficace pour l'absorption globale de deux oligo-éléments (bore, manganèse) dans nos essais en sols carencés à $\mathrm{pH}$ normal $(a ̀$ 6,0) et en absence de virus (Fig. 3). On a également vu une tendance à l'efficacité d'absorption du phosphore chez les blés les plus tolérants au virus, comme le Maringa et la lignée 98HF-181. D'autres données obtenues dans divers environnements supportent l'hypothèse qu'une partie des facteurs d'efficacité des racines sélectionnés par l'effet du BYD peut 


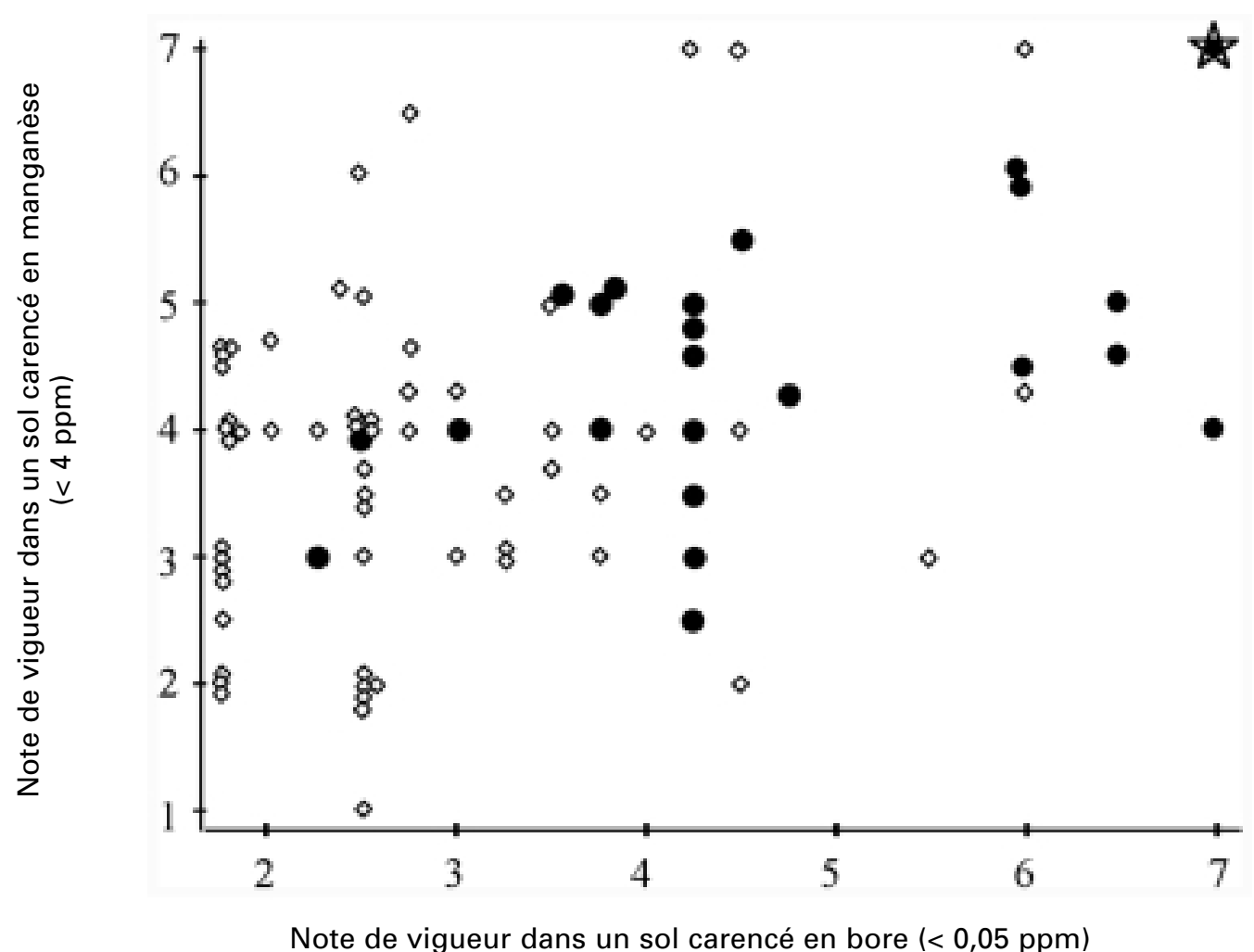

Figure 3. Vigueur de plusieurs lignées de blé et de triticale dans des essais d'aptitude à croître en sols carencés en bore ou en manganèse. Les notes élevées sont les meilleures. Le symbole choisi réfère à la réaction déjà obtenue dans des essais en sols non carencés, sous inoculation artificielle de virus (BYD). Cercles pleins : lignées tolérantes au virus; losanges vides : blés sensibles au virus; étoile : lignée Mu2EL6Hat, classée parmi les plus tolérantes au virus.

également aider à la tolérance aux carences, à l'hypoxie et aux pourritures des racines. Nos résultats suggèrent donc qu'un gain de tolérance au virus pourrait provenir du cumul de plusieurs mécanismes d'efficacité des racines, ce qui se traduit également par un gain de tolérance à d'autres stress relatifs aux racines.

Notons ici une difficulté. L'étude des carences est compliquée par le fait que les graines peuvent contenir des réserves substantielles d'oligo-éléments. Chez le blé, le grain issu de plantes cultivées en sol riche peut contenir des réserves de manganèse qui combleraient la moitié des besoins de la plante. II faut donc effectuer au moins deux cycles consécutifs en sol carencé pour évaluer adéquatement l'efficacité de la plante ainsi que les effets de la carence sur la physiologie et la résistance aux stress (Longnecker et al. 1991).

Un exemple de réussite de l'approche systémique est observable au Brésil. Avant l'agriculture moderne, les sols brésiliens étaient souvent lessivés, carencés, et le climat était propice au développement de multiples maladies fongiques, bactériennes et virales, et à l'apparition de plusieurs insectes et nématodes. Avec tous ces stress simultanés, c'est peut-être au Brésil que le germoplasme de blé a rencontré historiquement les conditions les plus stressantes. C'est donc dans un contexte systémique de stress multiples que la phytogénétique brésilienne a fait un progrès simul- tané sur plusieurs fronts tout en conservant des interactions et des systèmes de régulation efficaces du point de vue énergétique. Dans nos travaux en phytogénétique, nous avons constaté que le germoplasme brésilien est le plus intéressant, car il intègre la tolérance aux virus, aux sols carencés, à l'hypoxie, à la sécheresse, et aux maladies des racines, tout en ayant un bon potentiel de rendement. Notre recherche démontre qu'on peut incorporer ce progrès dans le germoplasme canadien, à condition d'opérer également dans un contexte de stress complexes, dans le cadre d'une approche systémique où le BYD et I'hypoxie jouent un rôle important. On alterne entre les stress simples et les stress complexes dans les processus de sélection dans le but de conserver à la fois la tolérance, la résistance et la plasticité de réponse aux stress.

Toutes ces observations ouvrent la porte à nombre de recherches cartésiennes, spécialisées, utiles, mais qui ne peuvent se substituer aux approches systémiques et plus globales. Dans la pratique, le terrain offre toujours des situations complexes et différentes à chaque année. II est donc logique que nos pratiques de sélection combinent régulièrement plusieurs stress à la fois, et ce, dans divers environnements pour évaluer la plasticité des facteurs de tolérance.

Les liens inattendus entre tolérance aux virus, tolérance à l'hypoxie et efficacité d'absorption des minéraux ont sûrement des fondements biochi- 
miques. Si on examine de plus près les sentiers métaboliques où les oligo-éléments jouent un rôle primordial, on trouve que ces minéraux peuvent affecter les mécanismes de résistance, la formation de lignine, le pouvoir allélopathique et les voies de la régulation de la résistance systémique acquise. En effet, le manganèse est important dès l'entrée dans la voie du shikimate, et joue un rôle parfois avec le cuivre et le bore dans les sentiers de la synthèse phénolique (Graham et Webb 1991; fig. 2). De plus, les mécanismes de résistance systémique acquise (RSA), les protéines PR et les phytoalexines sont aussi dépendants du manganèse. Par ailleurs, les racines peuvent être arrêtées net dans leur croissance par une mince couche de sol déficient en manganèse (Webb et Dell 1990). Chez le blé, la carence en manganèse est augmentée par plusieurs facteurs environnementaux, et prédispose à diverses maladies (Huber et McCay-Buis 1993). Or, on a constaté que la sélection du germoplasme sous inoculation virale aide parfois à identifier des génotypes efficaces dans l'absorption du manganèse. Les racines de tels génotypes sont aptes à traverser les zones carencées et à puiser des nutriments dans des horizons plus étendus. Soulignons enfin que la molécule glyphosate obtient son action herbicide par inhibition de la voie du shikimate, avec comme résultat que les plantes touchées peuvent devenir plus sensibles aux agents pathogènes des racines (Descalzo et al. 1996; Lévesque et Rahe 1992) et aux carences en manganèse.

Les racines de certaines plantes excrètent des composés appelés sidérophores, particulièrement essentiels en sols pauvres (Romheld et Marschner 1990). L'absorption des oligo-éléments dépend surtout de pompes ioniques et d'enzymes dont certaines sont spécifiques et d'autres moins (Hays 1993; Marchner 1995). Plusieurs enzymes sont associées à l'entrée du calcium, du cuivre, du manganèse, du zinc, du magnésium et du potassium. Tous ces mécanismes dépensent de l'énergie (Geiger et Servaites 1991) et doivent être bien régulés pour offrir un bilan positif à la plante.

Le mouvement des minéraux dans le film d'eau qui entoure les particules du sol s'apparente à une situation de chromatographie sur couche mince. Le déficit hydrique qui amincit le film d'eau affecte de manière différentielle la vitesse de migration et de séparation de divers ions, ce qui se traduit par l'apparition de carences spécifiques (Barber 1974). D'autre part, la stimulation du métabolisme provoquée par une fertilisation azotée surabondante peut créer des besoins en minéraux qui dépassent la capacité d'absorption des racines, favorisant également l'apparition de carences spécifiques. Finalement, même si certains effets des carences sont simples, d'autres peuvent être plus complexes. Par exemple, la plante carencée en bore montre d'abord un ralentissement ou un arrêt de croissance des racines qui affecte l'ensemble de sa croissance (Abdel Nasser et Abdel Aal 2002). Par la suite, une carence généralisée se développe puisque les racines ne sont plus assez vigoureuses. Cette réduction du développement des racines aggrave la sensibilité aux stress d'excès d'eau, de sécheresse et de maladies. C'est alors que les facteurs de causalité deviennent difficiles à préciser, et que divers experts auront partiellement raison en attribuant les pertes à des causes différentes.

La quantité de photosynthétats dirigés vers les racines est beaucoup plus abondante que la biomasse des racines ne le révèle (Lambers 1987). Une partie des sucres est utilisée pour la sécrétion d'exsudats de diverses natures qui contribuent à alimenter les microorganismes de la rhizosphère. Ces derniers offrent souvent des bénéfices à la plante en protégeant la racine contre les agents pathogènes, en fixant l'azote, ou en aidant à dissoudre et à transporter les minéraux comme le phosphate et le manganèse (Dommergues et Mangenot 1970). D'autres exsudats sont directement destinés à la dissolution de composés minéraux. Par ailleurs, pour fonctionner normalement, les racines consomment beaucoup de sucres par respiration. Les maladies, nématodes et insectes consomment une partie de la biomasse de racines et en réduisent la croissance et la performance de diverses manières. L'hypoxie est un stress qui force la plante à utiliser la voie des pentoses pour le métabolisme des racines (Kozlowski 1984) à moins que l'aérenchyme et/ou la leghémoglobine des racines (Hill 1998) n'offrent une alternative plus efficace. Or, la voie des pentoses peut avoir un coût énergétique environ 20 fois plus grand que le cycle de Krebs qui opère en conditions optimales. Sous un climat pluvieux, on ne peut sous-estimer l'importance économique de la tolérance à l'hypoxie, facteur de stress dont les effets se manifestent après moins de $24 \mathrm{~h}$ chez les plantes sensibles (Kozlowski 1984). II est donc important de sélectionner des plantes qui conservent une stabilité dans leur efficacité énergétique, car le potentiel de consommation d'énergie par les racines est très grand. Les racines étant exposées à des interactions génotypes $x$ environnements $(G \times E)$ importantes, cela pose un grand défi aux phytogénéticiens et aux agronomes.

\section{Approches statistiques}

L'efficacité de la plante à gérer ses photosynthétats de manière efficace est donc cruciale, tel qu'expliqué par Wallace et Yan (1998) dans un livre qui aborde les fondements de la génétique quantitative. Les coûts physiologiques des mécanismes de résistance et de tolérance font entièrement partie du problème du phytogénéticien qui s'occupe de caractères quantitatifs, lesquels incluent la plupart des paramètres agronomiques et plusieurs mécanismes de résistance aux stress. Des liens existent pourtant entre les paramètres observés au niveau aérien et ceux qui concernent le sol et les racines. Quand on veut étudier plusieurs paramètres à la fois, on en arrive rapidement à des problèmes d'analyses statistiques. II n'est pas facile de comprendre et de démontrer comme significatives toutes les relations complexes qui existent entre les divers paramètres du végétal à travers plusieurs environnements. Or, ces relations sont obligatoirement présentes, puisque tous les sentiers métaboliques sont en compétition les uns contre les autres pour l'usage des mêmes photosynthétats. II faut alors rappeler le verdict du statisticien Nester (1996) : les statistiques ne doivent jamais remplacer le raisonnement. La meilleure étude initiale de cas complexes avec plusieurs paramètres, sites, années, espèces et cultivars passe par les approches multi- 


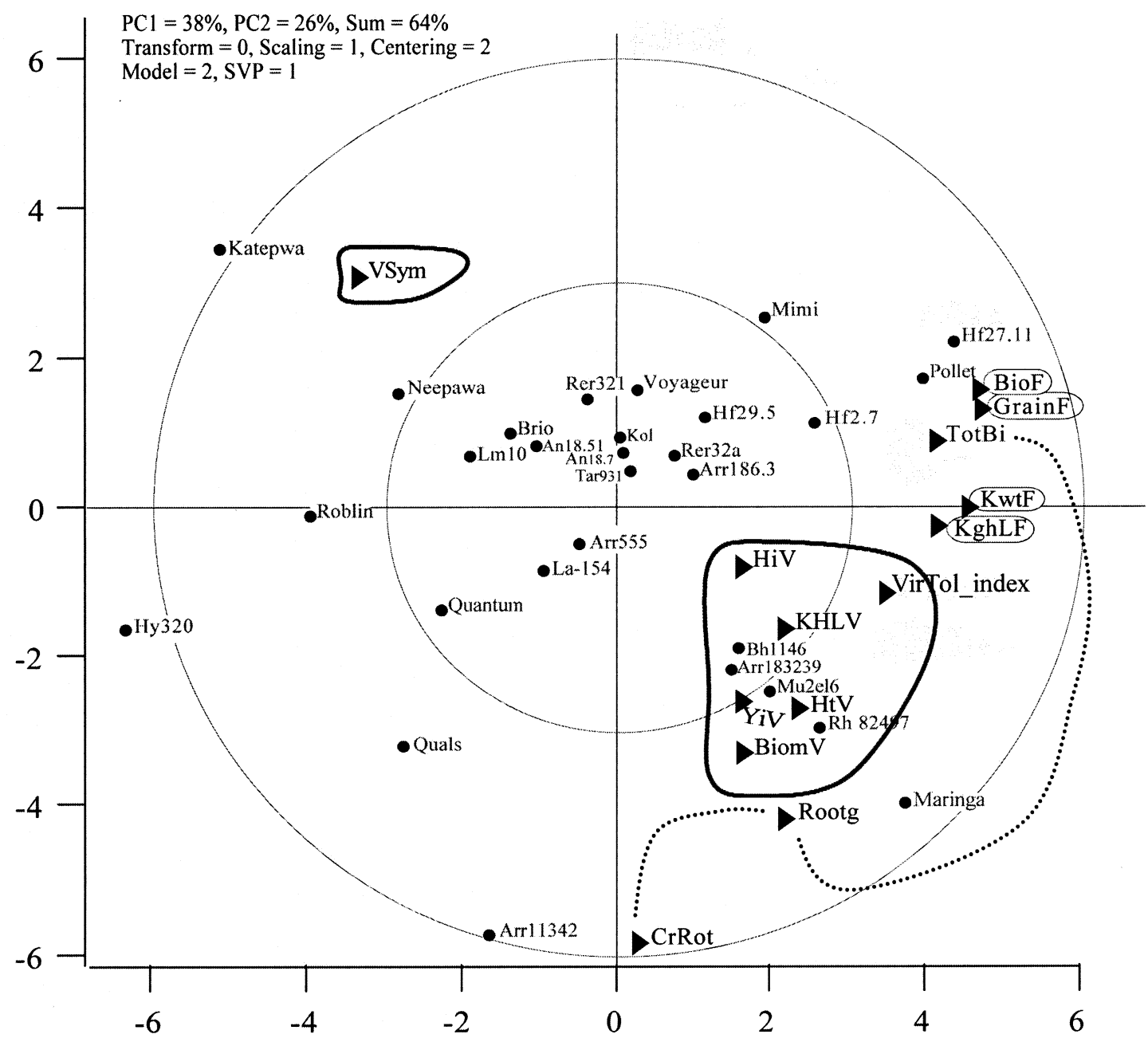

Figure 4. Relations entre lignées de blé et plusieurs paramètres, présentées par le logiciel GGE Biplot, pour des essais sur le comportement de lignées de blé dans des essais de tolérance au BYD, à l'excès d'eau au champ, et à l'hypoxie au niveau des racines en serres. Les cercles pleins correspondent aux lignées de blé, et les triangles aux paramètres. Les paramètres évalués sous pression de virus étaient la hauteur des plantes ( $\mathrm{HtV}$ ), la biomasse aérienne (biomV), le rendement (YiV), l'indice de récolte (HiV), les symptômes visuels (Vsym), le poids spécifique (KHLV), et l'indice global de tolérance VirTollndex (paramètres dans les zones encerclées, vers le bas à droite et en haut à gauche). Les paramètres évalués en serres sous hypoxie incluent la biomasse totale, la biomasse des racines, et la pourriture du collet (respectivement, TotBi, Rootg et CrRot, reliés par la ligne pointillée). Les paramètres évalués sous excès d'eau au champ incluent la biomasse, le rendement en grains, le calibre des grains et leur poids spécifique (respectivement, BioF, GrainF, KwtF et KghLF, noms encerclés à droite).

paramétriques, assistées par des logiciels appropriés. Pour ces objectifs, le logiciel DataDesk® (Data Description Inc., Ithaca, NY, USA) offre un premier aperçu intéressant, et le logiciel GGE Biplot donne une des méthodes les plus efficaces pour étudier simultanément les effets complexes des environnements stressants et la valeur des génotypes (Yan et Tinker 2005). Les regroupements possibles grâce à ce logiciel aident également à faire de meilleurs choix parmi les paramètres, environnements et lignées étudiés, si on veut s'orienter pertinemment vers des approches cartésiennes pour l'étude approfondie de certaines relations observées.

Un exemple d'utilisation du logiciel GGE Biplot est présenté pour montrer comment cet outil peut offrir une vision nouvelle de l'effet de stress divers comme le BYD, I'excès d'eau au champ et l'hypoxie au niveau des racines en serres. Les lignées ou paramètres qui sont à la fois loin du centre et rapprochés les uns des autres sont corrélés positivement; les lignées et paramètres opposés (par rapport au centre des axes canoniques $\mathrm{PC} 1$ et $\mathrm{PC} 2$ ) sont en corrélation négative.

Pour plusieurs paramètres associés à la tolérance, les réactions à l'excès d'eau au champ et au BYD sont associées dans le graphique obtenu avec GGE Biplot (Fig. 4). L'indice global de tolérance au virus (VirTollndex, indice pondéré compilé à base de données normalisées des paramètres et apte à corriger contre le lien entre la hauteur excessive et la tolérance au virus) (Comeau et Haber 2004), semble associé aux facteurs de qualité du grain sous excès d'eau au champ (KwtF et KghLF). Enfin, la mesure de biomasse totale en serres en présence d'hypoxie des racines (TotBi) s'avère associée au rendement en 
grains sous excès d'eau au champ, alors que la biomasse de racines sous ces mêmes conditions en serre (Rootg) est plutôt associée à la biomasse, au rendement et à la hauteur mesurés au champ sous stress de virus (BiomV, YiV et HtV). Les lignées de blé Maringa, Rh82497 et Mu2EL6 sont parmi celles qui ont la plus grande abondance de racines sous hypoxie en serre. Or, la lignée Mu2EL6 est la source directe de la sélection Mu2EL6Hat, dont on a souligné l'efficacité exceptionnelle dans l'usage du bore et du manganèse (Fig. 3).

II est important de mentionner que trois lignées (Maringa, Rh82497 et Mu2EL6) ont amené certains problèmes en tant que parents, à cause de leur tardiveté qui est toujours très difficile à dissocier des tolérances. L'observation répétée du lien entre tardiveté et tolérance confirme une importante proposition de Wallace et Yan (1998) : les progrès dans une direction sont souvent amenuisés par un recul pour $d$ 'autres paramètres. C'est pourquoi les projets axés sur un nombre insuffisant de paramètres sont voués à l'échec quant au potentiel d'applications pratiques. Le Dr Wallace (comm. pers.) nous avait mentionné que l'écueil de la tardiveté était fréquent et apparaissait aussi dans d'autres espèces comme le haricot, quand on essayait d'améliorer la résistance aux maladies des racines. La lignée Mu2EL6Hat, mentionnée précédemment, est par ailleurs une sélection plus hâtive obtenue à partir de la lignée Mu2EL6, et elle offre sans doute un meilleur point de départ en tant que source de gènes de tolérance. Le logiciel GGE Biplot est donc utile d'une part pour aider à mieux voir les liens entre paramètres, mais aussi pour identifier plus rapidement les lignées qui se distinguent dans un environnement stressant tout en faisant exception à ces corrélations nuisibles qui compliquent la tâche du phytogénéticien (Yan et Tinker 2005).

\section{CONCLUSION}

On ne doit pas entrer dans un domaine de recherche de ce genre sans faire l'effort d'intégrer les contributions de toutes les disciplines utiles. Et pour justifier I'usage inclusif de tant de sciences et même de philosophie, citons le philosophe Edgar Morin (1986): "On se rend encore difficilement compte que la disjonction et le morcellement des connaissances affectent, non seulement la possibilité d'une connaissance de la connaissance, mais aussi nos possibilités de connaissance sur nous-mêmes et sur le monde, provoquant ce que Gusdorf appelle une "pathologie du savoir" ". Ce survol rapide des approches scientifiques et philosophiques à un problème complexe ouvre la voie à d'autres considérations plus pratiques. II existe un grand nombre d'éléments stressants qui peuvent interagir avec les racines des plantes. Ces facteurs peuvent interagir entre eux de manière linéaire ou chaotique pour offrir une diversité d'environnements si vaste qu'il devient impossible de comprendre la totalité du problème par l'approche cartésienne. Dès lors, il faut mettre l'emphase voulue sur la notion d'efficacité physiologique, car la résistance n'a rien de magique, et son coût énergétique, variable dans chaque environnement donné, fait partie de l'équation. L'approche systé- mique nous enseigne également qu'il faut étudier les stress dans un système complexe qui s'apparente le plus possible à la nature, ou dans le cas qui nous concerne, à l'agroécosystème. Nous avons sans doute innové en allant résolument vers des applications pratiques, en imposant simultanément plusieurs facteurs de stress aux populations de blé, et en comparant le résultat aux effets de plusieurs situations naturelles de stress. L'approche systémique a mené au développement d'un processus de croisements et de sélection génétique sous stress, dont les résultats sont des plus prometteurs. Les progrès découlant de cette approche sont une affaire à suivre, tant pour la tolérance aux carences que pour la tolérance aux maladies des racines. C'est la synthèse et l'aboutissement pratique d'une manière nouvelle de percevoir le fonctionnement du vivant.

\section{REMERCIEMENTS}

Merci au Dr V. R. Caetano qui nous a beaucoup guidés de par sa propre expérience vers une compréhension globale des espèces étudiées, à André Brunelle et Denis Pageau pour avoir fourni les sols carencés et de bons conseils, à Jean Collin et René Paquet pour plusieurs années de support dans nos travaux, et à Luc Couture et Lucien Bordeleau pour leurs bons conseils scientifiques.

\section{RÉFÉRENCES}

Abdel Nasser, L.E. et A.E. Abdel Aal. 2002. Effect of boron deficiency on some physiological and biochemical aspects during the developmental stages of wheat (Triticum aestivum L.) plant. OnLine J. Biol. Sci. 2 : 470-476.

Barber, S.A. 1974. Influence of the plant root on ion movement in the soil. Pages 525-564 in E.W. Carson (éd.), The plant root and its environment. University Press of Virginia. Charlottesville, VA, USA.

Brown, J.K.M. 2002. Yield penalties of disease resistance in crops. Curr. Opin. Plant Biol. 5 : 339-344.

Browning, J.A. 1998. One phytopathologist's growth through IPM to holistic plant health. Annu. Rev. Phytopathol. $36: 1-24$

Brunelle, A. 2005. Production écologique et rentable de céréales à paille : aspects agronomiques. Pages 55-60 in Colloque céréales : des cultures aux premiers rangs. Cahier de conférences, CRAAQ, Sainte-Foy, Québec.

Comeau, A. et S. Haber. 2002. Breeding for BYDV tolerance in wheat as a basis for a multiple stress tolerance strategy. Pages 82-92 in M. Henry et A. McNab (éds.), Barley yellow dwarf disease, recent advances and future strategies. CIMMYT, Mexico.

Comeau, A. et S. Haber. 2004. Resistance and tolerance to BYDVs/CYDVs in cereals. Pages 204-218 in $\mathrm{H}$. Lapierre et A. Signoret (éds.), Viruses and virus diseases of Poaceae (Gramineae). INRA, Paris.

Comeau A., V. Caetano, C.A. St-Pierre et S. Haber. 2001. Accelerated breeding: dream or reality? Pages 671-679 in Z. Bedo et L. Lang (éds.), Wheat in a global environment. Kluwer Academic Publishers, Dordrecht, The Netherlands.

Cruz, P.J., F.I.F. de Carvalho, V.R. Caetano, S.A. Silva, A.J. Kurek et R.L. Barbieri. 2001. Traits associated with lodging resistance in bread wheat. Cienc. Rural $31: 563-568$ 
Descalzo, R.C., Z.K. Punja, C.A. Lévesque et J.E. Rahe. 1996. Identification and role of Pythium species as glyphosate synergists on beans (Phaseolus vulgaris) grown in different soils. Mycol. Res. $100: 971-978$.

Dommergues, Y. et F. Mangenot. 1970. Écologie microbienne du sol. Masson et Cie, Paris. 796 pp.

Geiger, D. et J. Servaites. 1991. Carbon allocation and response to stress. Pages 103-127 in H. Mooney, W. Winner, E. Pell et E. Chu (éds.), Response of plant to multiple stresses. Academic Press, San Diego.

Graham, R.D. et M.J. Webb. 1991. Micronutrients and resistance and tolerance to disease. Pages 329-370 in J.J. Mortwedt, F.R. Cox, L.M. Shuman et R.M. Welch (éds.), Micronutrients in agriculture. Second Edition. Madison, WI, USA: Soil Science Society of America.

Hays, S.M. 1993. Secrets of soil nutrient uptake. Agric. Res. $41: 18-19$.

Higginbotham, R.W., T.C. Paulitz et K.K. Kidwell. 2004. Evaluation of adapted wheat cultivars for tolerance to pythium root rot. Plant Dis. 88 : 1027-1032.

Hill, R.D. 1998. What are hemoglobins doing in plants? Can. J. Bot. $76: 707-712$.

Huber, D.M. et T.S. McCay-Buis. 1993. A multiple component analysis of the take-all disease of cereals. Plant Dis. 77 : 437-447.

Kozlowski, T.T. 1984. Flooding and plant growth. Academic Press, Orlando.

Lambers, H. 1987. Growth, respiration, exudation and symbiotic associations: the fate of carbon translocated to the roots. Pages 125-145 in P.J. Gregory, V.A. Lake et D.A. Rose (éds.), Root development and function. Soc. Exp. Biol. Seminar Series 30. Cambridge University Press, Cambridge, UK.

Lemerle, D., B. Verbeek, R.D. Cousens et N.E. Coombes. 1996. The potential for selecting wheat varieties strongly competitive against weeds. Weed Res. 36 : 505-513.

Lévesque, A. et J.E. Rahe. 1992. Herbicide interactions with fungal root pathogens, with special reference to glyphosate. Annu. Rev. Phytopathol. 30 : 579-602.

Lewontin, R.C. 1991. Biology as ideology: the doctrine of DNA. HarperPerennial, New York. 112 pp.

Lewontin, R.C. 2000. The triple helix: gene, organism, and environment. Harvard University Press, Cambridge, MA. $136 \mathrm{pp}$.

Lewontin, R. 2001. It ain't necessarily so: the dream of the human genome and other illusions. $2^{\text {nd }}$ Ed. New York Review of Books. $330 \mathrm{pp}$.

Lewontin, R.C., S. Rose et L.J. Kamin. 1984. Not in our genes: biology, ideology and human nature. Penguin, Harmondsworth, UK.
Longnecker, N.E., N.E. Marcar et R.D. Graham. 1991. Increased manganese content of barley seeds can increase grain yield in manganese-deficient conditions. Austr. J. Agric. Res. 42 : 1065-1074.

MacKey, J. 1973. The wheat root. Pages 827-842 in E.R. Sears et L.M.S. Sears (éds.), Proc. 4th Intl. Wheat Genet. Symp. Missouri Agr. Exp. Stn., Columbia, MO, USA.

MacKey, J. 1984. Assimilation and yield structure - a plant breeder's conclusion. Sver. Utsädesför. Tidskr. 94 : 135-144.

Marchner, H. 1995. Mineral nutrition of higher plants. $2^{\text {nd }} E d$. Academic Press, New York. 889 pp.

Miralles, D.J., G.A. Slafer et V.R. Lynch. 1997. Rooting patterns in near-isogenic lines of spring wheat for dwarfism. Plant Soil $197:$ : 79-86.

Morin, E. 1986. La méthode. Vol. 3. La connaissance de la connaissance. Seuil, Paris. 255 pp.

Morin, E. 1999. La tête bien faite. Seuil, Paris. 153 pp.

Nester, M.R. 1996. An applied statistician's creed. Appl. Stat. $45: 401-410$.

Nicol, J., S.A. Bagci, H. Hekimhan, B. Tunali, N. Bolat, H.J. Braun et R. Trethowan. 2004. Strategy for the identification and breeding of resistance to dryland root rot complex for International spring and winter wheat breeding programs. Poster paper in "New directions for a diverse planet". Proc. 4th Intl. Crop Sci. Congr., Brisbane, Australia. www.cropscience.org.au.

Nodichao, L. 1997. Biodiversité racinaire et résistance à la sécheresse. Mémoire de maîtrise. Université Laval, Québec. 168 pp.

Romheld, V. et H. Marschner. 1990. Genotypical differences among graminaceous species in release of phytosiderophores and uptake of iron phytosiderophores. Pages 77-83 in N. El Bassam, M. Dambroth et B.C. Loughman (éds.), Genetic aspects of plant mineral nutrition. Kluwer Academic Publishers, Dordrecht, The Netherlands.

Wallace, D.H. et W. Yan. 1998. Plant breeding and wholesystem crop physiology - improving crop maturity, adaptation, and yield. CAB International, New York, NY, USA. $390 \mathrm{pp}$

Webb, A.J. et Dell, B. 1990. Effect of manganese supply on development of wheat (Triticum aestivum) roots. Pages 235-239 in M.L. Van Beusichem (éd.), Plant nutrition physiology and applications. Kluwer Academic Publishers, Dordrecht, The Netherlands.

Yan, W. et Tinker, NA. 2005. An integrated biplot analysis system for displaying, interpreting and exploring genotype x environment interaction. Crop Sci. 45 : 1004-1016. 